\title{
Heavy flavor measurements at the STAR experiment
}

\author{
Pavol Federic ${ }^{1, \star}$ \\ ${ }^{1}$ Nuclear Physics Institute of the Czech Academy of Sciences, Rez near Prague, Czech Republic
}

\begin{abstract}
In ultra-relativistic heavy-ion collisions at RHIC, a new state of nuclear matter with extreme properties is produced - the strongly interacting quark-gluon plasma. Heavy quarks, predominantly produced at early stages of collisions owing to their large masses, provide an exceptional probe for exploring the hot and dense medium created in such collisions. The Heavy Flavor Tracker and Muon Telescope Detector have been fully operational since 2014 and have significantly improved STAR's capabilities in measuring both open and hidden heavy flavor hadrons in heavy-ion collisions. We present an overview of recent heavy flavor results obtained at the STAR experiment.
\end{abstract}

\section{Introduction}

Heavy flavor quarks are predominantly produced early in heavy-ion collisions in hard scattering processes. They experience the full evolution of the system. These features make heavy quarks ideal probes for studying the properties of the strongly coupled Quark-Gluon Plasma (sQGP) at RHIC. Two new detectors dedicated to heavy flavor measurements, the Heavy Flavor Tracker (HFT) [1] and the Muon Telescope Detector (MTD) [2], were fully installed at the STAR experiment in the beginning of 2014. In this proceedings we will report recent results from STAR using the HFT and MTD.

\section{STAR experiment}

STAR is a large-acceptance, multi-purpose detector at RHIC. The main subsystems used in the following analyses are the Time Projection Chamber (TPC), Time Of Flight (TOF) detector, HFT and MTD.

The TPC reconstructs track trajectories, measures their momenta and provides particle identification via measuring ionization energy losses $(\mathrm{dE} / \mathrm{dx})$. The TOF greatly improves the identification capabilities, especially in low $p_{\mathrm{T}}$ region, through measuring the time-of-flight of charged particles.

The HFT consists of 3 sub-detector systems, the Silicon Pixel (PXL) detector utilizing the state-ofthe-art Monolithic Active Pixel Sensor (MAPS), Intermediate Silicon Tracker (IST) and Silicon Strip Detector (SSD). The PXL detector is made of two layers of MAPS. The low material budget of the PXL detector (0.4-0.6\% radiation length per layer) in combination with excellent position resolution provides precise measurements of track impact parameters. The track impact parameter resolution for charged kaons exceeds the requirement of less than $55 \mu \mathrm{m}$ at transverse momentum $\left(p_{\mathrm{T}}\right)$ equal to 750 $\mathrm{MeV} / \mathrm{c}$.

\footnotetext{
^e-mail: pavolfederic@gmail.com
} 
The MTD is made of multi-gap resistive plate chambers placed at the outermost layer of STAR behind the solenoidal magnet, which is used as a hadron absorber. It is designed for muon triggering and identification with timing resolution $\sim 100 \mathrm{ps}$ for $p_{\mathrm{T}}>1.2 \mathrm{GeV} / \mathrm{c}$. It has a geometrical acceptance of $45 \%$ in azimuth within $|\eta|<0.5$.

\section{Open heavy flavor measurements}

The total charm production cross sections at mid-rapidity $(|\eta|<1)$ have been measured by STAR in $\mathrm{p}+\mathrm{p}$ and $\mathrm{Au}+\mathrm{Au}$ collisions at $\sqrt{s_{N N}}=200 \mathrm{GeV}$, and are found to scale with the number of binary nucleon+nucleon collisions [4]. This observation supports the expectation that heavy flavor quarks are mainly produced in initial hard parton scatterings at RHIC. Relatively long lifetimes of heavy flavor quarks enable them to experience the whole evolution of the system. Therefore they can serve as calibrated probes to study QGP.

Shown in figure 1 is the $D^{0}$ nuclear modification factor $\left(R_{A A}\right)$ in $0-10 \% \mathrm{Au}+\mathrm{Au}$ collisions at $\sqrt{s_{N N}}$ $=200 \mathrm{GeV}$ extracted from about 780 million Minimum Bias (MB) events with the HFT (black solid circles). Also shown are the STAR published results from 1.1 billion MB events recorded in 2010 and 2011 without the HFT (red open circles) [4]. The vertical bars (brackets) represent statistical (systematic) uncertainties, while the grey shaded areas indicate the systematic uncertainties from $\mathrm{p}+\mathrm{p}$ reference for the $R_{A A}$ obtained from the HFT data. The latter were not present for the published results due to cancellation of efficiency corrections between the $\mathrm{p}+\mathrm{p}$ and $\mathrm{Au}+\mathrm{Au}$ data. The boxes around unity denote the overall normalization uncertainties in the $\mathrm{Au}+\mathrm{Au}$ and $\mathrm{p}+\mathrm{p}$ data, respectively. The new $R_{A A}$ measurement confirms the strong suppression of $D^{0}$ yields at high transverse momenta, which is comparable to the suppression observed for light hadrons and indicates strong charm interactions with the medium.

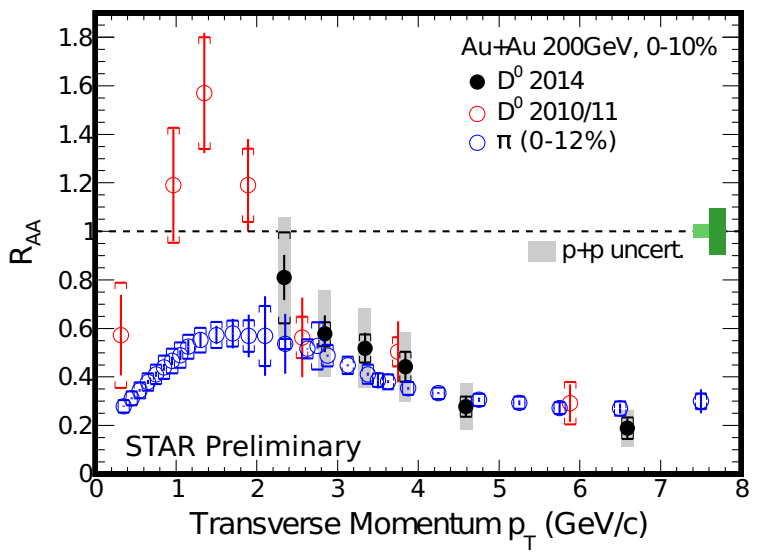

Figure 1. Measured $D^{0} R_{A A}$ for $0-10 \%$ central $\mathrm{Au}+\mathrm{Au}$ collisions with (solid circles) and without (open circle) the HFT. The open blue circles show $\pi R_{A A}$ [3] for comparison.

The HFT has also enabled the first measurements of elliptic flow $\left(v_{2}\right)$ for open charm hadrons at RHIC. Significant non-zero $D^{0} v_{2}$ is shown in the left panel of figure 2 for $p_{\mathrm{T}}>2 \mathrm{GeV} / \mathrm{c}$. The vertical bars (brackets) represent statistical (systematic) uncertainties, while the grey shaded areas show the estimate of contributions from non-flow effects. Shown in the right panel is the $D^{0} v_{2} / n_{q}$ as function 
of $\left(\sqrt{m_{0}^{2}+p_{\mathrm{T}}^{2}}-m_{0}\right) / n_{q}$, where $m_{0}$ is the hadron mass and $n_{q}$ the number of constituent quarks. The $D^{0}$ $v_{2} / n_{q}$ is below those of light hadrons [5], but still consistent within uncertainties, suggesting non-zero charm quark flow.
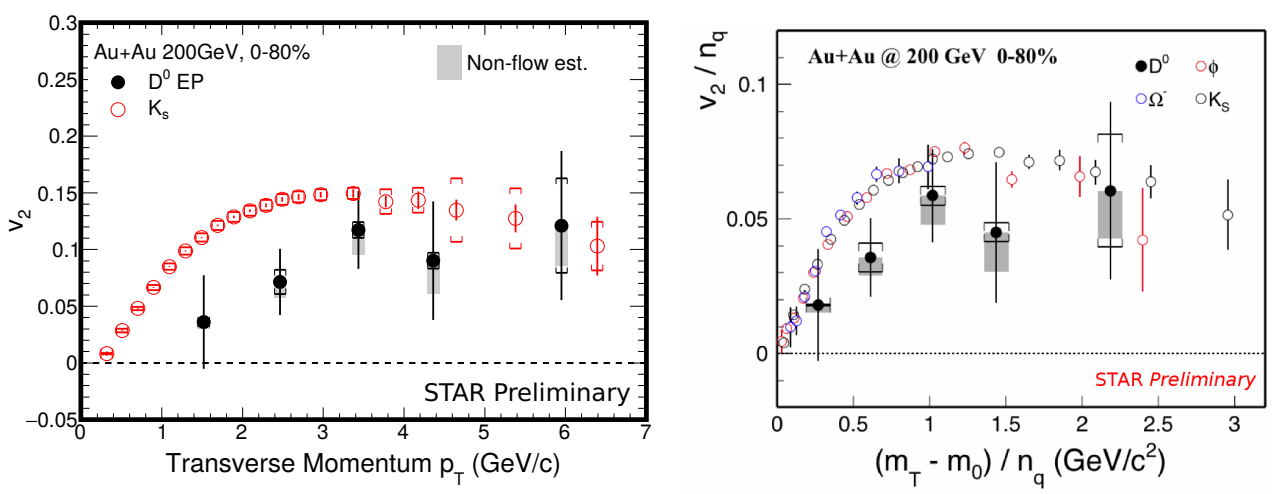

Figure 2. Left: measured $D^{0} v_{2}$ compared to that of light hadrons; Right: $v_{2} / n_{q}$ as function of $\left(\sqrt{m_{0}^{2}+p_{\mathrm{T}}^{2}}-m_{0}\right) / n_{q}$ for $D^{0}$ and other particle species.

The measurements of $D^{0} R_{A A}$ and $v_{2}$ are compared to theoretical calculations. As seen in figure 3, the TAMU [6] and SUBATECH [7] models describe both the $D^{0} R_{A A}$ and $v_{2}$. The DUKE [8] model describes the $R_{A A}$ well but underestimates the $v_{2}$. These models use different approaches to describe interactions of charm quarks in the medium and allow to extract transport coefficients. The TAMU model uses a non-perturbative T-matrix approach with potentials obtained from Lattice QCD calculations to describe two-body interactions as a function of the transferred 4-momentum. The SUBATECH group employs a pQCD approach with the Hard Thermal Loop approximation for soft collisions. The charm quark diffusion coefficient $2 \pi T D_{s}$ extracted from the TAMU (SUBATECH) model ranges between 2- 10 (2-4) for $\mathrm{T} / \mathrm{T}_{\mathrm{c}}=1-2$. The DUKE group uses a generalized Langevin approach with transport properties tuned to LHC data, i.e. $2 \pi T D_{s}=7$. Figure 4 shows the diffusion coefficient extracted from different model calculations [7, 9-14] compared to the yellow band inferred from the STAR data, which are consistent with the lattice QCD calculations $[13,14]$ within large uncertainties.

\section{Quarkonium measurements with the MTD}

While the heavy quark production can be calculated by $\mathrm{pQCD}$, the evolution into quarkonia from quark-antiquark pair is a non-perturbative process. Precise measurements of quarkonium production in $\mathrm{p}+\mathrm{p}$ collisions can help to understand quarkonium production mechanisms. The situation in heavyion collisions is more complicated. Quarkonia may dissociate owing to the color screening of the heavy quark-antiquark potential by the QGP. Such a dissociation depends on the temperature of the QGP and varies for different quarkonium states of different binding energies. On the other hand, cold nuclear matter effects (CNM), such as shadowing/anti-shadowing of parton distribution functions, initial-state parton energy loss and final-state nuclear absorption, along with regeneration via coalescence of deconfined charm quarks may also play a role. Therefore quarkonium production can be used to constrain the QGP temperature once other effects are understood. 

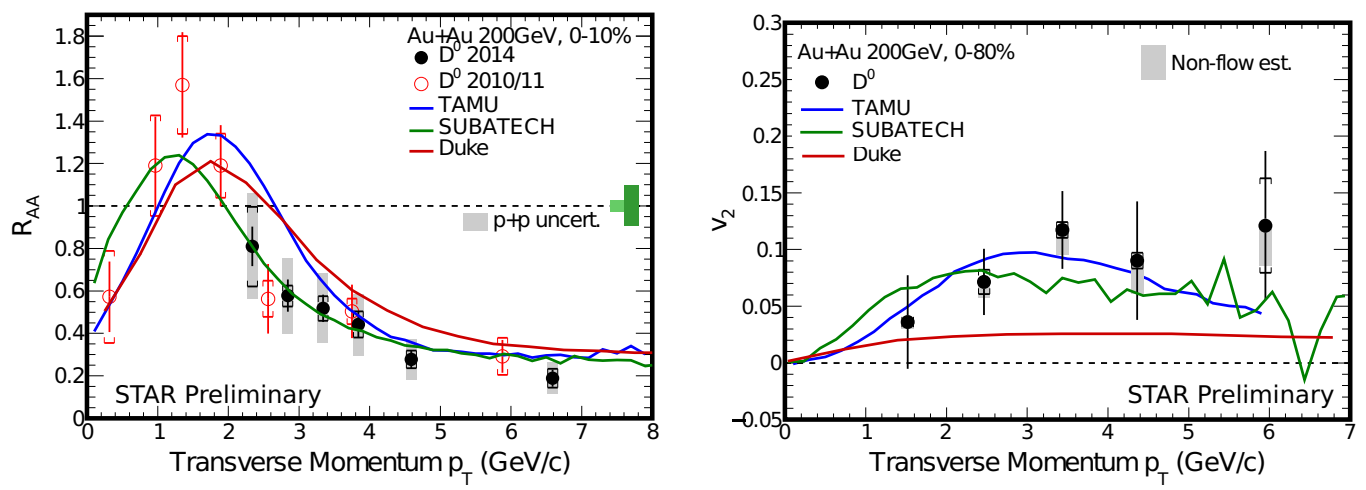

Figure 3. Left: $D^{0} R_{A A}$ in $0-10 \%$ centrality compared to model calculations; Right: $D^{0} v_{2}$ in $0-80 \%$ centrality compared to model calculations.

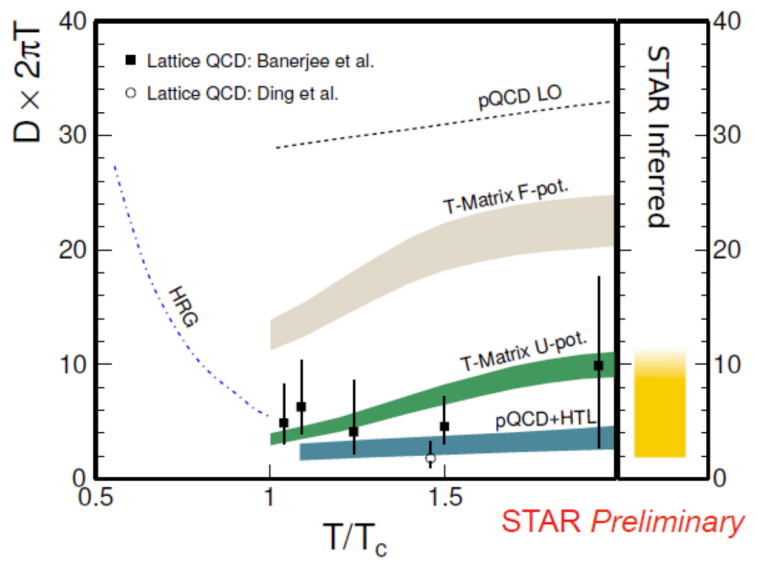

Figure 4. Charm quark diffusion coefficient from model calculations and the inferred range from STAR measurements. See text for details.

Figure 5 shows the results on $\mathrm{J} / \psi R_{A A}$ in $\mathrm{Au}+\mathrm{Au}$ collisions at $\sqrt{s_{N N}}=200 \mathrm{GeV}$ in the di-electron and in di-muon channels for different collision centralities. The new MTD results from the di-muon decay channel are consistent with the di-electron channel results $[15,16]$ within uncertainties. The dependence of $\mathrm{J} / \psi R_{A A}$ on the number of participating nucleons $\left(N_{\text {part }}\right)$ has also been measured. The left (right) panel of figure 6 shows the abovementioned di-muon results (red stars) for $p_{\mathrm{T}}>0$ (5) $\mathrm{GeV} / \mathrm{c}$ compared to the results from PHENIX [17] in Au+Au collisions at the same centre-of-mass energy (black open circles) as well as the measurements from ALICE [18] and CMS [19] in $\mathrm{Pb}+\mathrm{Pb}$ collisions at $\sqrt{s_{N N}}=2.76 \mathrm{TeV}$ (blue squares). For $p_{\mathrm{T}}>0 \mathrm{GeV} / \mathrm{c}$, the STAR results are consistent with the PHENIX results, showing a decreasing $R_{A A}$ towards more central collisions. On the other hand, the ALICE results are almost independent of centrality and significantly less suppressed in central collisions compared to STAR results. Such a difference could be explained by larger regeneration 


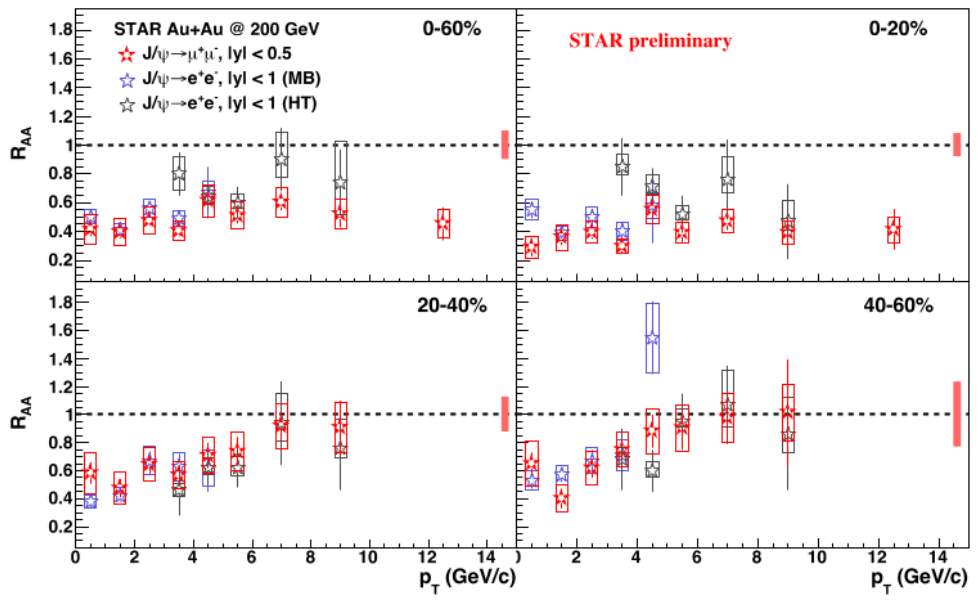

Figure 5. $\mathrm{J} / \psi R_{A A}$ in $\mathrm{Au}+\mathrm{Au}$ collisions at $\sqrt{s_{N N}}=200 \mathrm{GeV}$ in four centrality classes.

contributions to low $p_{\mathrm{T}} \mathrm{J} / \psi$ at the LHC with much higher $c \bar{c}$ yields. At high $p_{\mathrm{T}}$, the $R_{A A}$ at both RHIC and LHC have a decreasing trend towards more central collisions, and the LHC results are lower than those at RHIC. This is likely due to higher temperature reached at the LHC which leads to stronger J/ $\psi$ dissociation. Two different transport models [20, 21], both including dissociation and regeneration, can qualitatively describe the measured $R_{A A}$. However, there seems to be a tension between the model calculations and experimental data at high $p_{\mathrm{T}}$.
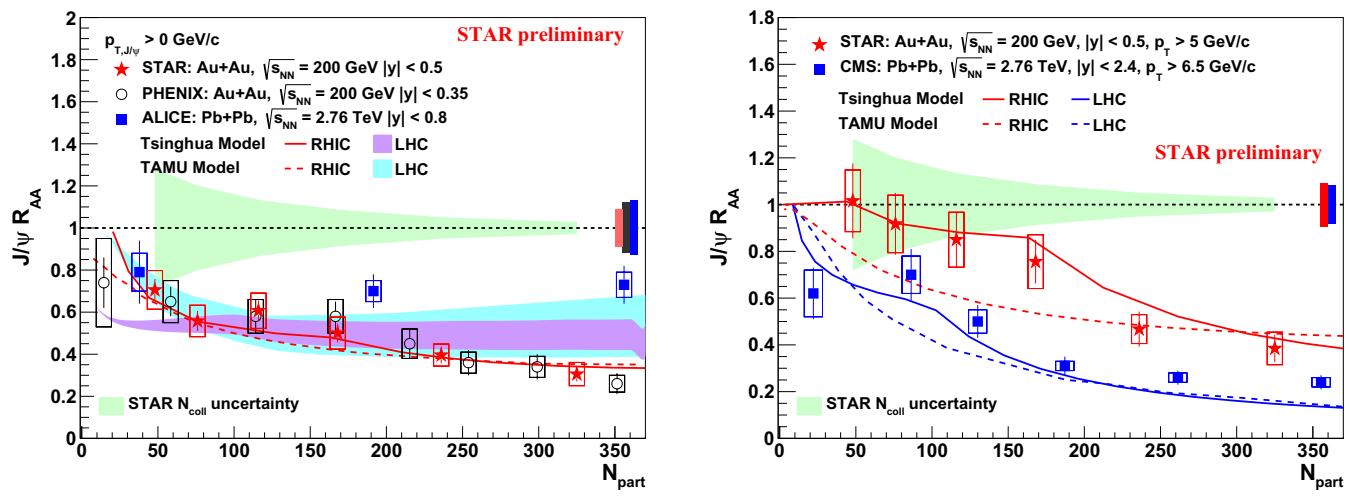

Figure 6. Left: $\mathrm{J} / \psi R_{A A}$ for $p_{\mathrm{T}}>0 \mathrm{GeV} / \mathrm{c}$ in $\mathrm{Au}+\mathrm{Au}(\mathrm{Pb}+\mathrm{Pb})$ collisions at $\sqrt{s_{N N}}=200 \mathrm{GeV}(2.76 \mathrm{TeV})$; Right: $\mathrm{J} / \psi R_{A A}$ for $p_{\mathrm{T}}>5(6.5) \mathrm{GeV} / \mathrm{c}$ in $\mathrm{Au}+\mathrm{Au}(\mathrm{Pb}+\mathrm{Pb})$ collisions at $\sqrt{s_{N N}}=200 \mathrm{GeV}(2.76 \mathrm{TeV})$. The vertical bars (boxes) represent statistical (systematic) uncertainties. See text for details.

Shown in the left panel of figure 7 is the reconstructed $\Upsilon$ signal from the di-muon decay channel using the same MTD data described above. Compared to the di-electron channel, the di-muon channel has the advantage of better invariant mass resolution, thanks to the significantly suppressed bremsstrahlung radiation. The unlike-sign (red points) spectrum is fitted to the sum of $\Upsilon(1 \mathrm{~S}), \Upsilon(2 \mathrm{~S})$ 
and $\Upsilon(3 S)$ signal distributions, $B \bar{B}$, Drell-Yan and combinatorial background distributions. The shapes of the signal distributions are Gaussian functions with means fixed to the PDG values, and widths determined from detector simulations, while the shape of $B \bar{B}$ and Drell-Yan background is determined from PYTHIA. Both the shape and normalization of the combinatorial background are obtained by fitting the like-sign (black points) spectrum. Shown in the right panel are the ratio of the excited $\Upsilon$ state yield with respect to the ground state yield, obtained from the abovementioned fit, the worldwide $\mathrm{p}+\mathrm{p}$ data and CMS measurements $[22,23]$ in $\mathrm{Pb}+\mathrm{Pb}$ collisions at $\sqrt{s_{N N}}=2.76 \mathrm{TeV}$. There is an indication of larger $\Upsilon(2 \mathrm{~S}+3 \mathrm{~S}) / \Upsilon(1 \mathrm{~S})$ ratio at RHIC than that at the LHC.
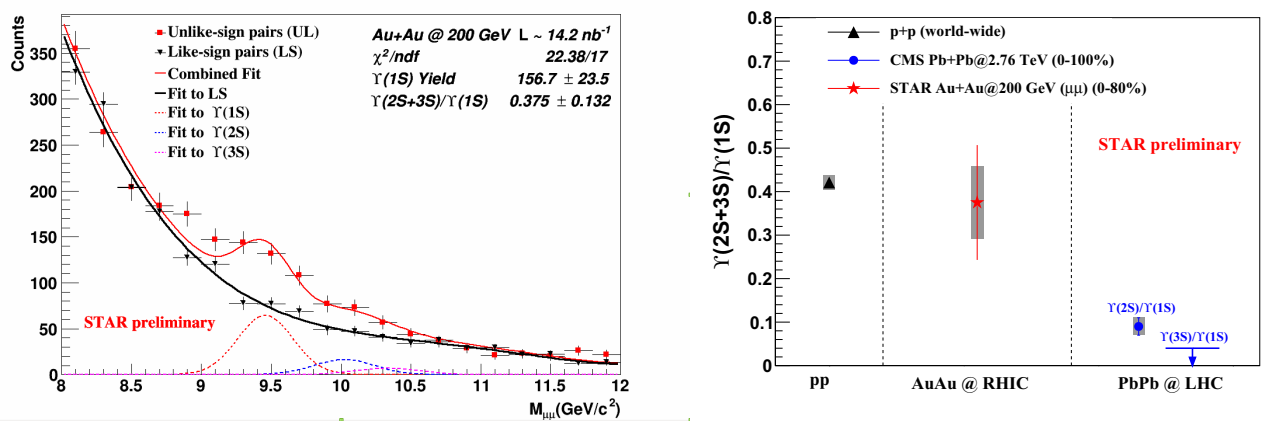

Figure 7. Left: invariant mass spectra of di-muon pairs in $\mathrm{Au}+\mathrm{Au}$ collisions at $\sqrt{s_{N N}}=200 \mathrm{GeV}$; Right: ratio of excited $\Upsilon$ states to the ground state measured in $\mathrm{p}+\mathrm{p}$ collisions, $\mathrm{Au}+\mathrm{Au}$ collisions at $\sqrt{s_{N N}}=200 \mathrm{GeV}$ and $\mathrm{Pb}+\mathrm{Pb}$ collisions at $\sqrt{s_{N N}}=2.76 \mathrm{TeV}$. See text for details.

\section{Summary}

STAR has carried out the first measurements with the recently installed HFT and MTD in Au+Au collisions. The measurements of $D^{0} R_{A A}$ and $v_{2}$ indicate strong interactions between charm quarks and the medium created in heavy-ion collisions. Model calculations which can simultaneously describe the $R_{A A}$ and $v_{2}$ give a range of charm quark diffusion coefficients between 2 and $\sim 12$ for the QGP temperature between $\mathrm{T}_{c}$ and $2 \mathrm{~T}_{c}$. The measurements of $\mathrm{J} / \psi$ with the MTD via the di-muon channel are consistent with the di-electron channel results and show a distinct rising trend in the $R_{A A}$ with transverse momentum in 20-60\% centrality. The suppression observed at high $p_{\mathrm{T}}$ in $0-20 \%$ central collisions is consistent with dissociation due to color screening. The measurements of high- $p_{\mathrm{T}} \mathrm{J} / \psi$ $R_{A A}$ and ratio of yields of different $\Upsilon$ states indicate a lower medium temperature reached at RHIC than at LHC.

\section{Acknowledgements}

The work has been supported by the grant 13-20841S of the Czech Science Foundation (GACR). The author's participation in the conference was supported by grant INGO LG14004207 of MSMT of the Czech Republic. 


\section{References}

[1] Technical Design Report: The STAR Heavy Flavor Tracker (2011)

[2] L. Ruan et al., J. Phys. G: Nucl. Part. Phys. 36, 095001 (2009)

[3] B. I. Abelev et al., Phys. Lett. B 655, 104 (2007)

[4] L. Adamczyk et al. (STAR Collaboration), Phys. Rev. Lett. 113, 142301 (2014)

[5] B. I. Abelev et al. (STAR Collaboration), Phys. Rev. C 77, 054901 (2008)

[6] M. He, R. J. Fries and R. Rapp, Phys. Rev. C 86, 014903 (2012)

[7] M. Nahrgang et. al., Phys. Rev. C 91, 014904 (2015)

[8] W. Zha et. al., Phys. Rev. C 88, 067901 (2013)

[9] A. Andronic et. al., The Eur. Phys. J. C 76(3), 107 (2016)

[10] O. Kaczmarek, Nuclear Physics A 931, 633 (2014)

[11] F. Riek and R. Rapp, Phys. Rev. C 82, 035201 (2010)

[12] B. Svetitsky, Phys. Rev. D 37, 248 (1998)

[13] H. T. Ding et. al., Phys. Rev. D 86, 014509 (2012)

[14] D. Banerjee, S. Datta, R. Gavai and P. Majumdar, Phys. Rev. D 85, 014510 (2012)

[15] L. Adamczyk et. al. (STAR Collaboration), Phys. Lett. B 722, 55 (2013)

[16] L. Adamczyk et. al. (STAR Collaboration), Phys. Rev. C 90, 024906 (2014)

[17] A. Adare et al. (PHENIX collaboration), Phys. Rev. D 82, 012001 (2010)

[18] B. B. Abelev et al. (ALICE Collaboration), Phys. Lett. B 734, 314 (2014)

[19] S. Chatrchyan et al. (CMS Collaboration), JHEP 05, 063 (2012)

[20] Y. Liu et. al., Phys. Let. B 67872 (2009). K. Zhou et. al., Phys. Rev. C 89, 054911 (2014)

[21] X. Zhao and R. Rapp, Phys. Rev. C 82, 064905 (2010)

[22] W. Zha et. al., Phys. Rev. C 88, 067901 (2013)

[23] S. Chatrchyan et al. (CMS Collaboration), PRL 109, 222301 (2012) 